$M$ ammillaria xaltianguensis fue encontrada por Sánchez Mejorada al Norte de Xaltianguis, municipio de Acapulco, estado de Guerrero. También ha sido colectada cerca de Tierra Colorada, en los márgenes del Río Omitlán y a la orilla de la Presa de La Venta, de este mismo estado. En Michoacán, se encuentra en El Salto, municipio de Aguililla, Barranca Honda, (Pilbeam, 1999) y entre Playa Azul y Arteaga (Sánchez-MejoradaR, 1973; Bravo-Hollis y Sánchez-Mejorada, 1991; Pilbeam, 1999; Hunt, 2006).

En la descripción original, SánchezMejorada-R. (1973), señala que un ejemplar colectado en el estado de Jalisco y cultivado en el Jardín Botánico de Huntington, pudiera referirse a este taxón. Sin embargo, en el inventario para la entidad, Arreola-Nava (1990) no la considero a esta especie por no hallarse colectas en los herbarios nacionales. En Las Cactáceas de México, Bravo-Hollis y Sánchez-Mejorada (1991) no citan a Jalisco como parte de la distribución de la especie, pero Pilbeam (1999) menciona su presencia en el estado, con una localidad dudosa, por lo que puede tratarse de lo citado por Sánchez-MejoradaR. (1973). Sin embargo, Anderson (2001), Guzmán et al. (2003) y Hunt (2006) no refieren su existencia en Jalisco, por lo que este trabajo confirma su presencia en la entidad.

En el mes de diciembre del 2009 , durante un recorrido de trabajo por el municipio de Cuautitlan de García Barragán, Jalisco, el primer autor encontró una cactácea del género Mammillaria la que fue identificada con la bibliografía disponible (BravoHollis y Sánchez-Mejorada, 1991; Hunt, 2006) y la revisión de ejemplares del Herbario Nacional de México (MEXU) como Mammillaria xaltianguensis Sánchez-Mej.

\title{
MAMMILLARIA XALTIANGUENSIS (CACTACEAe) EN Jalisco
}

\author{
Jesús Padilla-Lepe ${ }^{1,4}$, Hilda Julieta Arreola-Nava ${ }^{2}$ \\ y LUIS GUZMÁN-HERNÁNDEZ ${ }^{3}$
}

${ }^{1}$ Egresado del Centro Universitario de Ciencias Biológicas y Agropecuarias. Universidad de Guadalajara

${ }^{2}$ Departamento de Botánica y Zoología del Centro Universitario de Ciencias Biológicas y Agropecuarias. Universidad de Guadalajara

${ }^{3}$ Laboratorio de Botánica, Herbario ZEA, Departamento de Ecología y Recursos

Naturales, del Centro Universitario Costa Sur. Universidad de Guadalajara

${ }^{4}$ Autor para la correspondencia: ludtita@yahoo.com

Se encontró al Sur del poblado El Chico, antes del entronque que va a Chacala y Sehuaya cercana a los márgenes del Río Chacala a una altitud de 400 m s.n.m. Se trata de una planta de habito rupícola, (Figura, 1A) preferentemente crece en laderas de exposición Este dentro del bosque tropical subcaducifolio, acompañada de Acanthocereus occidentalis, Bursera sp., Ceiba pentandra, Ficus sp., Pachycereus pecten-aboriginum, Passiflora sp., Peniocereus cuixmalensis, Peperomia sp., Pseudobombax ellipticum y Opuntia puberula entre otras.

Otras poblaciones encontradas se localizan al Sureste de Ahuacapan, municipio de Autlán de Navarro, y en La Calera, municipio de Casimiro Castillo, a una altitud que va desde 900 a 1,300 m s.n.m. Habitan en bosque tropical caducifolio en ecotonía con bosque tropical subcaducifolio; aunque también se ha encontrado con elementos de bosque de Quercus en las partes más altas, estas poblaciones fueron identificadas erróneamente y citadas como Mammillaria supraflu- men en Flora de Manantlán (VázquezG. et al., 1995).

Mammillaria xaltianguensis en la Norma Oficial Mexicana, NOM059-SEMARNAT-2010 (SEMARNAT, 2010) tiene la categoría de Sujeta a Protección Especial. De acuerdo al criterio uno del método de evaluación del riesgo de extinción de plantas (MER) (SEMARNAT, 2010), que toma en cuenta el área de distribución de las poblaciones del taxón, es probable que al ampliarse la distribución de la especie, modifique su categoría de riesgo, sin embargo no es el propósito de este trabajo y otros criterios deberán ser analizados.

Las poblaciones en el municipio de Autlán de Navarro y Casimiro Castillo son poco abundantes y de difícil acceso, pero están dentro de la Reserva de la Biosfera Sierra de Manantlán, por lo que de alguna forma están protegidas. Sin embargo, las poblaciones al sur de Jalisco están a un costado de la carretera en construcción de Cuautitlán a Manzanillo y su hábitat está sien- 

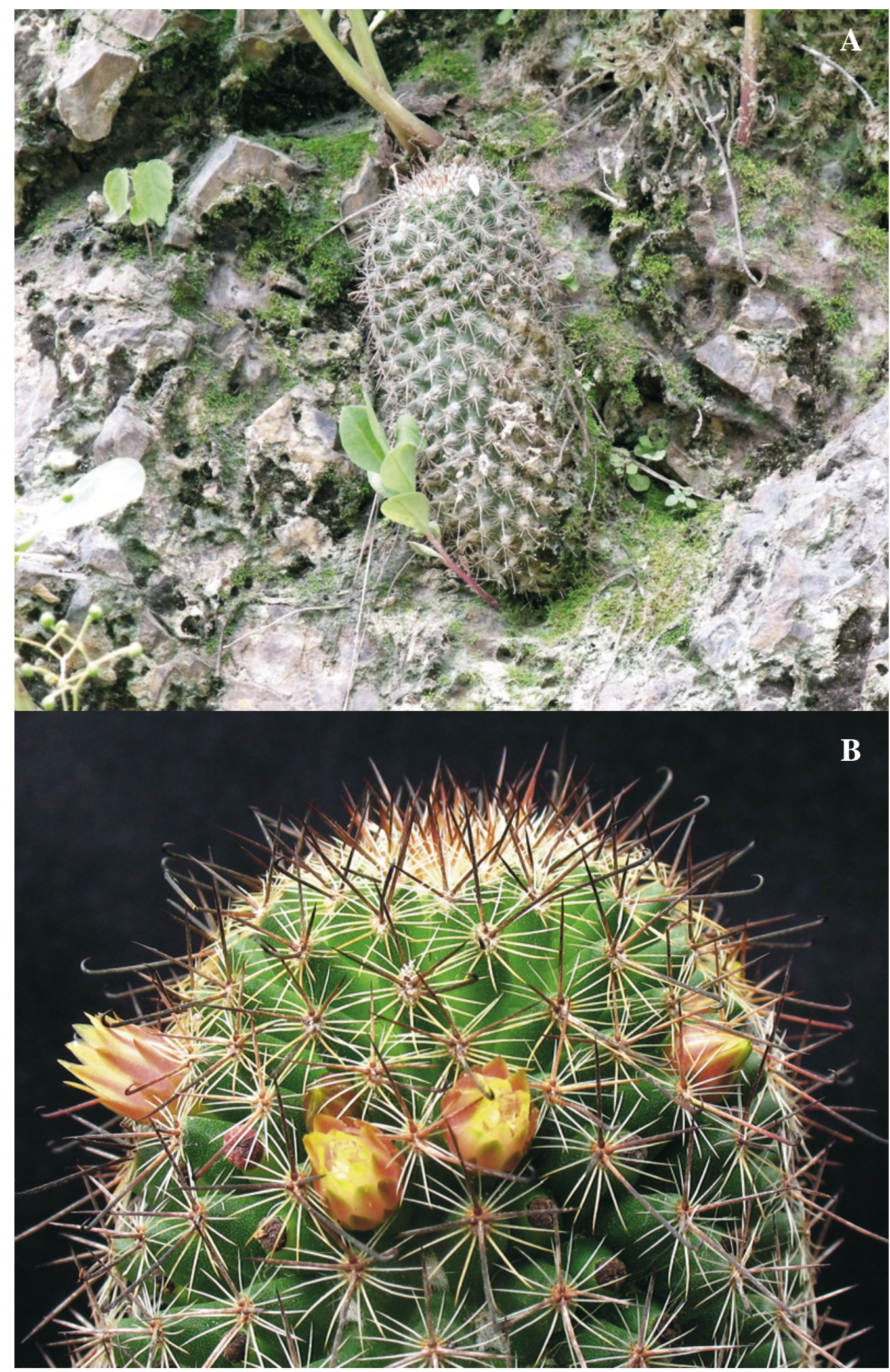

Figura 1. A. Mammillaria xaltianguensis en la localidad de El Chico, Cuautitlan de García Barragán, Jalisco. B. Detalles de M. xaltianguensis, el cual florece de julio a agosto. 


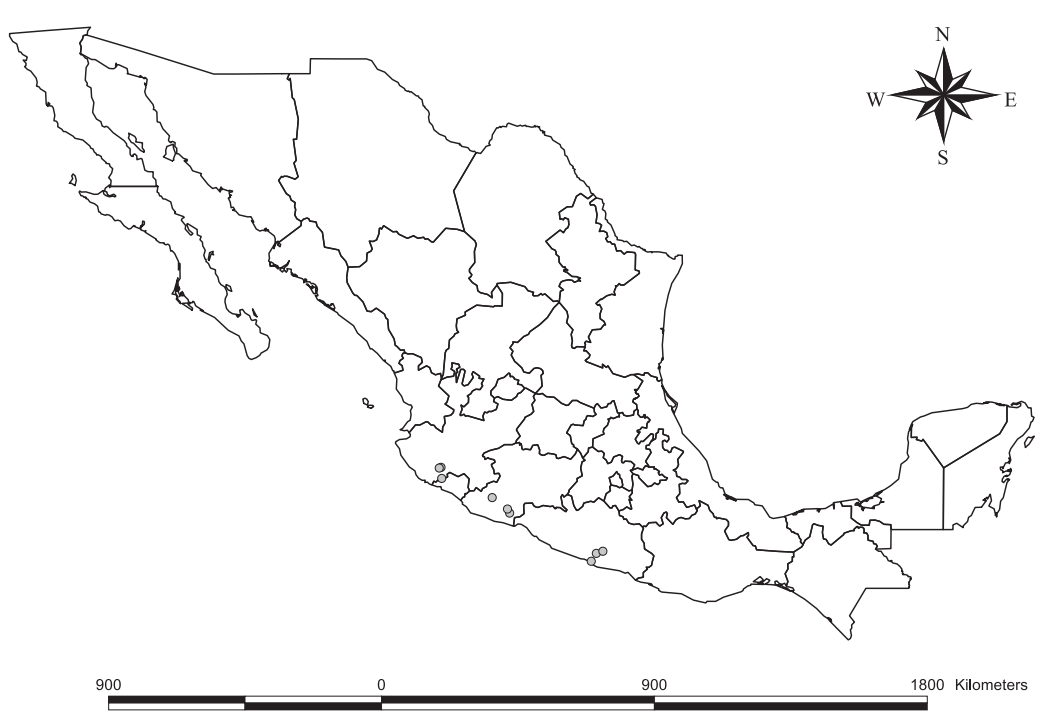

Figura 2. Distribución de Mammillaria xaltianguensis (॰) en México.

do destruido. Además de lo anterior Mammillaria xaltianguensis, como muchas cactáceas más, es endémica de México por lo que se debe proteger su hábitat y generarse programas locales de conservación para esta especie. En el Centro Universitario de Ciencias Biológicas y Agropecuarias (CUCBA), Universidad de Guadalajara, se ha iniciado un programa para la propagación de esta especie a partir de semilla con fines de conservación para su eventual reintroducción y disposición para jardines botánicos en intercambios.

La figura 2 muestra los puntos de distribución de la especie con base en los ejemplares de herbario y literatura consultada.

\section{Ejemplares examinados:}

MEXICO. GUERRERO: Acapulco, en Xaltianguis, a orillas del río del mismo nombre, creciendo sobre roca granítica a una altitud de 525 m. 1970, Hernando Sánchez Mejorada 70-0802, HOLOTIPO (MEXU); Tierra Colorada, cerros a la orilla de la Presa de La Venta, creciendo en grietas de roca caliza a una altitud $350 \mathrm{~m}$, Hernando Sánchez Mejorada. 1977, (MEXU). JALISCO, Cuautitlan de García Barragán, El
Chico, a orilla del Río Chacala, bosque tropical subcaducifolio. $400 \mathrm{~m}$. Coord. $19^{\circ} 20^{\prime} 04^{\prime \prime} \mathrm{N}, 104^{\circ} 17^{\prime} 40^{\prime \prime}$ O. Jesús Padilla-Lepe y H. J. ArreolaNava 202, 29 agosto 2010, (IBUG).

A continuación se presenta una descripción de las plantas encontradas, la cual no difiere mucho con respecto a la descripción original.

Plantas solitarias o en grupo. Tallos cilíndricos a subcilíndricos de hasta $20 \mathrm{~cm}$ de alto y 7-8 $\mathrm{cm}$ de diámetro, ápice no hundido, látex de color blanco, únicamente en la médula, raíces fibrosas. Tubérculos dispuestos en 8 y 13 series espiraladas, ligeramente tetra-angulados, de 6-7 $\mathrm{mm}$ de longitud, de 4-5 $\mathrm{mm}$ en la base de color verde claro. Axilas jóvenes, desnudas, después con tricomas cortos de color amarillento, cerdas de 1-8, blancas, tortuosas de hasta $12 \mathrm{~mm}$ de longitud. Aréolas pequeñas, redondeadas, las jóvenes con tricomas cortos de color amarillento, tempranamente caduco. Espinas radiales 16-17 subuladas, divergentes, rectas de color blanco o amarillento con la base marrón, de 3-4 $\mathrm{mm}$ de longitud. Espinas centrales cuatro, rectas con la base bulbosa, color moreno rojizo o amarillo ocre con puntas rojo moreno, ligeramente pubescentes, con el tiempo grisáceas, dis- puestas en forma de cruz, ascendentes, las superiores 5.5-6 $\mathrm{mm}$ de longitud, la inferior de 7-9 $\mathrm{mm}$ de longitud, rara vez ganchuda. Flores en corona cercanas a la mitad del tallo (Figura 1B), campanuladas de $13 \mathrm{~mm}$ de longitud; pericarpelo verde claro de $4 \mathrm{~mm}$ de diámetro; segmentos exteriores del perianto de 2-7 $\mathrm{mm}$ de longitud de 1-1.5 $\mathrm{mm}$ de ancho, romboidales de color rojizo, margen ligeramente dentado, de color amarillo claro; segmentos interiores del perianto de $6 \mathrm{~mm}$ de largo y $1.5 \mathrm{~mm}$ de ancho lanceolados, margen liso, translucidos de color amarillo con la parte superior verdosa; estambres escasos; filamentos delgados, blancos; anteras pequeñas de color amarillento; estilo de $7 \mathrm{~mm}$ de longitud, lóbulos del estigma cinco, de 1 $\mathrm{mm}$ de longitud de color amarillo pálido. Fruto inmaduro, ovoide de color verde obscuro de 4-5 mm de diámetro; pulpa pegajosa e incolora, perianto caduco, cuando madura es claviforme de color verde con la base rojiza de 16 $\mathrm{mm}$ de longitud y $6 \mathrm{~mm}$ de ancho esto sucede al año siguiente de la floración que lo origina. Semillas piriformes de $0.6-0.7 \mathrm{~mm}$ de ancho y $0.8-0.9 \mathrm{~mm}$ de largo de color café claro.

\section{Agradecimientos}

Se agradece a María Hilda Flores por su fina atención en MEXU, Claudia Elizabeth Rosales Contreras por la realización del mapa y a Raymundo Ramírez Delgadillo $\dagger$ por su ayuda en el herbario IBUG.

\section{Literatura citada}

Anderson E.F. 2001. The Cactus Family. Timber Press, Portland Oregon.

Arreola-Nava H. J. 1990. Inventario de las cactáceas de Jalisco y su distribución. Cactáceas y Suculentas Mexicanas 35: 3-12.

Bravo-Hollis H. y Sánchez-Mejorada H. 1991. Las Cactáceas de México. Vol. III. Universidad Nacional Autónoma de México, México, D.F.

Guzmán U., Arias S. y Dávila P. 2003. 
Catálogo de Cactáceas Mexicanas. Universidad Nacional Autónoma de México. Comisión Nacional para el Conocimiento y Uso de la Biodiversidad, México, D.F.

Hunt D. Ed. 2006. The New Cactus Lexicon. dh books, Milborne Port.

Pilbeam J. 1999. Mammillaria: The Cactus File Handbook 6. Cirio Publishing Services, Southampton.

Sánchez-Mejorada-R. H. 1973. Mammillaria xaltianguensis, una nueva espe- cie de Guerrero. Anales del Instituto de Biología 44:29-32.

SEMARNAT [Secretaria del Medio Ambiente y Recursos Naturales]. 2010. Norma Oficial Mexicana NOM059-SEMARNAT-2010, Protección ambiental - Especies nativas de México de Flora y Fauna Silvestres - Categorías de Riesgo y especificaciones para su inclusión, exclusión o cambio - Lista de Especies en Riesgo. Diario Oficial de la Federación 2a Sección, 30 de diciembre del 2010. <http://www.semarnat.gob.mx/leyesynormas/SEMARNAT \%20DOF/Norma\%20Oficial\%20 Mexicana\%20NOM-059-SEMARNAT-2010.pdf.

Vázquez-G. J. A., Cuevas-G. R., Cochrane T. S., Iltis H.H., Santana-M. F. J. y Guzmán-H. L. 1995. Flora de Manantlán: Plantas vasculares de la Reserva de la Biosfera Sierra de Manantlán JaliscoColima, México. Sida. Botanical Mis-

Recibido: 25 de febrero de 2011

Aceptado : 23 de julio de 2011 